\title{
ESTUDO DA VIABILIDADE DA APLICAÇÃO DE TÉCNICAS DE GERÊNCIA DO CONHECIMENTO NA EDUCAÇÃO TECNOLOGICA
}

\author{
Hertz Wilton de Castro Lins \\ Doutorando em Engenharia Elétrica e Computação pela Universidade Federal do Rio Grande do Norte \\ (UFRN), hertzw@gmail.com \\ Cláudio Rodrigues Muniz da Silva \\ Professor Dr. Departamento de Engenharia Elétrica da Universidade Federal do Rio Grande do Norte \\ (UFRN), claudio.rmsilva@gmail.com
}

\begin{abstract}
RESUMO
Este trabalho tem o objetivo de examinar conceitos e aspectos da Gerência do Conhecimento (GC) que possam contribuir no desenvolvimento de ferramentas mais adaptadas para os desafios atuais da Educação tecnológica (ET). A Gerência do Conhecimento pode ser vista, essencialmente, como uma disciplina que fornece subsídios para o projeto de processos que, ao longo de seus ciclos de vida, se beneficiam da extração formal automática do conhecimento utilizado nos mesmos. A idéia principal é a utilização deste conhecimento como base para um funcionamento otimizado no futuro. Um dos desafios da Educação tecnológica é a dificuldade de atualização dos conteúdos dos vários domínios de treinamento, resultante da rapidez com que as mudanças ocorrem em tudo que depende da tecnologia. A Gerência do conhecimento permite um tratamento formal e consistente de todas as etapas da educação tecnológica, viabilizando o desenvolvimento de ferramentas capazes de explorar o conhecimento adquirido para permitir um processo de adaptação mais flexível e dinâmico. Este artigo examina as formas e tecnologias envolvidas, mostrando sua viabilidade através de uma metodologia que demonstra como utilizar os conceitos relacionados com a GC no contexto da ET.
\end{abstract}

PALAVRAS-CHAVE: Educação Tecnológica, Gerência do Conhecimento, Modelagem do Conhecimento.

\section{FEASIBILITY STUDY OF APLICATION OF KNOWLEDGEMENT MANAGEMENT TECHNICS IN TECHNOLOGICAL EDUCATION}

\begin{abstract}
This study aims to examine concepts and aspects of the Knowledge Management (KM) which can contribute to development of tools more suited to the current challenges of education technology (ET). The Knowledge Management can be seen primarily as a discipline that provides grants for the design of processes over their life cycles, may benefit from formal automatic extraction of knowledge used in them. The main idea is to use this knowledge as a basis for optimal use in the future. One of the challenges of technological education is the difficulty of updating the content of the various areas of training, resulting from the speed with which changes take place in all that depends on the technology. The Knowledge Management allows a formal and consistent treatment of all stages of education technology, enabling the development of tools able to exploit the knowledge acquired to allow an adjustment process more flexible and dynamic. This article examines the ways and technologies involved, showing its feasibility through a possible methodology that demonstrates how to use the concepts related to KM in the context of ET.
\end{abstract}

KEY-WORDS: Technological Education, Knowledge Management, Knowledge Modeling. 


\section{ESTUDO DA VIABILIDADE DA APLICAÇÃO DE TÉCNICAS DE GERÊNCIA DO CONHECIMENTO NA EDUCAÇÃO TECNOLOGICA}

\section{INTRODUÇÃO}

As mudanças do mundo atual e principalmente seus avanços tecnológicos exigem das organizações cada vez mais melhorias no desempenho de seus negócios, no desempenho humano e na capacidade de inovação e adaptação. Neste contexto, elas estão desenvolvendo novas flexibilidades e descobrindo novas formas de explorar valores de seu conhecimento conseguindo, assim, benefícios como gerar vantagem competitiva, e por conseqüência, mais eficiência e produtividade no presente e no futuro [1] [2].

A Gerência do Conhecimento (GC) coordena a criação, transferência e aplicação de conhecimento, seja individual ou coletivo, nos processos de criação das organizações. Outro importante objetivo da GC é desenvolver estratégias e estruturas para retorno de recursos intelectuais e de informação [3] . Fornece também uma visão econômica do valor estratégico e organizacional focada na aquisição, compartilhamento e utilização do conhecimento na mesma [4], possui características interdisciplinares, que envolve pessoas, processos e metodologias no processo de criar, compartilhar, distribuir e aplicar o conhecimento. Como contribuições podem-se citar: sistemas de informação, tecnologia da informação, gestão estratégica, teoria organizacional, gestão de recursos humanos, ensino da ciência, psicologia cognitiva e inteligência artificial [5].

Entretanto, alguns cuidados devem ser tomados no contexto da implementação de soluções de GC, como, por exemplo, o foco que deve ser em pessoas e os negócios das organizações e não em tecnologias. Neste contexto; as metodologias para GC são responsáveis por disponibilizar a forma de trazer para um cenário prático os conceitos inerentes aos processos de manipulação do conhecimento como: criação, recuperação, atualização e aplicação do conhecimento.

Apesar de sua importância, não existe até o momento uma metodologia ou framework (que são mais genéricos que as metodologias) aceito em consenso pelos pesquisadores da área, por vários motivos, seja por não esclarecer os detalhes suficientes para sua implementação, seja por não abordar todos os requerimentos para uma efetiva e completa GC. Todavia o principal fator para a ausência deste método pode ser que estas metodologias sejam parte estratégica e sigilosa de planos de negócio e também propriedade intelectual [6] [7].

Este trabalho é motivado pela ausência de uma metodologia que forneça os detalhes para uma efetiva implementação de GC. É motivado também, em propor uma metodologia para GC que contribua com as atividades de composição e atualização dos conteúdos e conhecimentos envolvidos no processo de ensino tecnológico. Ensino este que exige qualificação e atualização de profissionais, novas habilidades, novos perfis e novos papeis de uma forma cada vez mais ágil, em virtude das mudanças no mundo, especificamente, as atualizações da tecnologia. [8] [9].

O artigo está organizado do seguinte modo: depois da introdução são apresentados os fundamentos do trabalho. Na seqüência a viabilidade de aplicação da GC na ET, seguidos da metodologia da pesquisa. O item seguinte estudo da viabilidade, demonstra a implementação de um processo através de um protótipo, em seguida a conclusão, e por fim as referencias bibliográficas.

\section{FUNDAMENTOS}

Nesta seção serão apresentados os conceitos fundamentais relacionados com a GC, tais como: 
dados, informação, conhecimento, tipos de conhecimento, processos, modelagem, metodologias, educação tecnológica e GC, objetos de conhecimento, visualização do conhecimento, e os conceitos e relações ente GC, Web semântica e educação.

\section{CONHECIMENTO}

Os dados podem ter vários tipos de definições dependendo do contexto de seu uso. No contexto da GC podem ser compreendidos como fatos que podem ser números, símbolos ou figuras sem contexto e interpretação [10]. As informações vez são baseadas em dados e acrescentam valor para a compreensão de um determinado assunto e, em contexto, são à base dos conhecimentos, segundo [11]. A informação tem as seguintes características: são transferíveis, transparentes, autônomas e mensuráveis. Conhecimento pode ser entendido como as associações funcionais e explicitas entre os itens de informação e/ou dados. Segundo [11], o conhecimento possui uma característica crucial que é a sua interiorização pelo conhecedor.

Na figura 1, a seguir, os conceitos e relações entre dados, informação e conhecimento são exemplificados. É apresentada uma pirâmide que ilustra estes conceitos a partir de um índice fictício do mercado de ações. Na primeira transição em (1), os caracteres: “ $\{5757,98$,$\} ” são$ transformados em dados: “57.571,98”, exemplificando o índice referente a determinado dia deste mercado. A transformação ocorre, com a aplicação de regras aos caracteres também chamada de sintaxe, permitindo assim dados serem manipulados por máquinas. A segunda transição em (2), dados para informação, ocorre quando existe a analise e interpretação dos dados. Considerando um determinado contexto, que no exemplo proposto seria a interpretação das variações do índice. A terceira transição, informação para conhecimento (3), ocorre com a compreensão da informação no contexto global através do entendimento e experiência acumulada de como a variação ou comportamento no tempo do índice, em questão, vai afetar o mercado como um todo. No item 2.2, a seguir, são apresentados os tipos de conhecimento e a sua respectiva importância no contexto de um ambiente de GC.

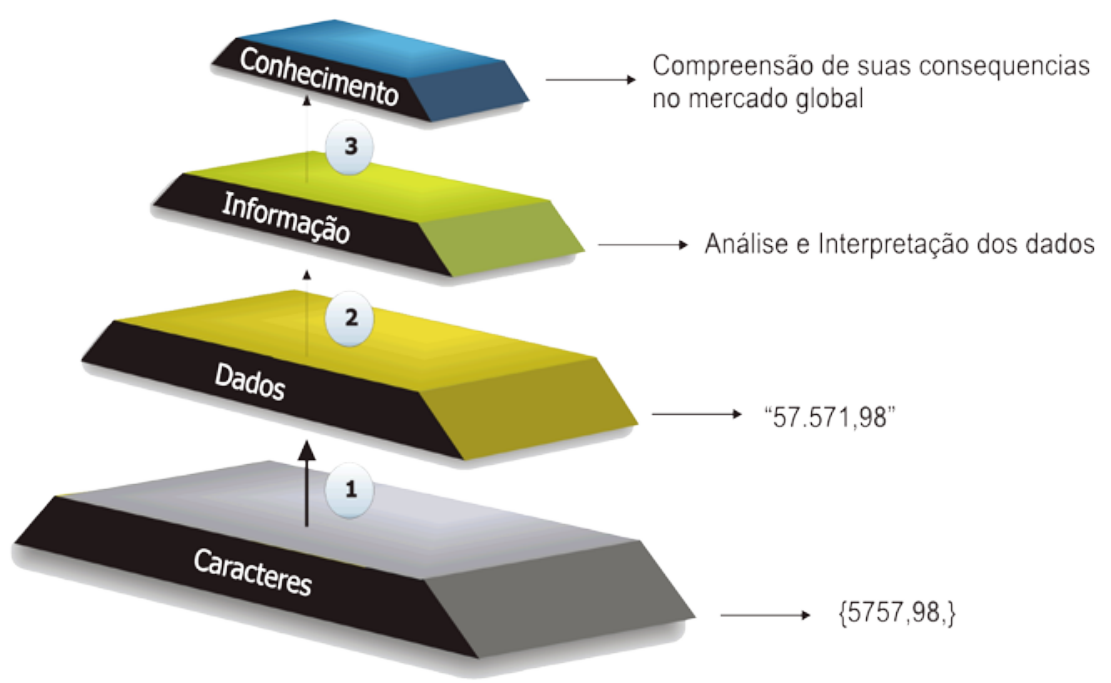

Figura 1 - Dados, informação e conhecimento.

\section{TIPOS DE CONHECIMENTO}

A importância do tipo do conhecimento, no contexto da GC, deve se ao fato do diferente tratamento que cada tipo receberá, segundo a literatura tradicional, o conhecimento pode ser a princípio de dois 
tipos: explicito e tácito [12]. O conhecimento explícito, segundo [10], é aquele que tem sido codificado tipicamente em objetos, palavras e números, e também na forma de gráficos, desenhos, especificações, manuais, procedimentos e podem, portanto, ser facilmente partilhados e entendidos. O conhecimento tácito, também chamado de conhecimento implícito, consiste dos modelos mentais, comportamentos e das perspectivas pessoais, em sua grande parte, é baseado na memória e experiência dos indivíduos. Este conhecimento é difícil de codificar, segundo [5], uma nova categorização é proposta em quatro níveis: o nível de conhecimento explicito que é composto por conhecimento tácito e explicito; o nível de alcance formado pelo conhecimento individual e coletivo; o nível de abstração que compreende conhecimento específico e conhecimento genérico; o nível de propósito que faz a distinção entre conhecimento declarativo e procedimental. Após a apresentação dos tipos de conhecimento o item 2.3, a seguir, concentra-se no conceito de modelagem e seu papel na GC.

\section{MODELAGEM}

A modelagem tem um papel crucial na GC, sua função é permitir a comunicação e colaboração entre os participantes do processo, sejam usuários ou sistemas. Para a realização dessa comunicação é necessário que ocorra previamente a definição e representação de conceitos relevantes e dos termos comuns, que precisam ser devidamente especificados formando o domínio do conhecimento. Como exemplo de abordagens de modelagem pode-se citar: as redes semânticas e os mapas do conhecimento [2] [13]. Alguns sistemas de GC exigem um nível de formalização do conhecimento que suporte operações como: interconexão entre sistemas, recuperação de informação, troca de informações e inferências, Nesta situação são utilizadas as ontologias que permitem a modelagem de detalhes como classes, atributos e relacionamentos, sua estrutura proporciona muito mais do que um vocabulário ou taxonomia permitindo a inclusão de conceitos abstratos e complexos formando assim um domínio de conhecimento com alto nível de significado [14]. O item 2.4, a seguir, apresenta o conceito de metodologia e traz algumas metodologias de GC encontradas na literatura da área.

\section{METODOLOGIAS}

Uma metodologia, que consiste de um conjunto de procedimentos a serem seguidos para alcançar um objetivo, é de extrema importância na GC porque ela será o guia para a implementação dos processos de conhecimento e será a responsável pela efetiva utilização do capital intelectual para incremento do desempenho da organização. Apesar de sua importância, não existe até o momento uma metodologia ou framework (que são mais genéricos que as metodologias) aceito em consenso pelos pesquisadores da área, por vários motivos, seja por não esclarecer os detalhes suficientes para sua implementação, seja por não abordar todos os requerimentos para uma efetiva e completa gerência do conhecimento. Mas o principal fator para a sua ausência pode ser que estas metodologias sejam parte estratégica e sigilosa de planos de negócio e também propriedade intelectual [6] [7] [15]. A seguir uma breve apresentação de algumas metodologias, e seus principais processos, presentes na literatura corrente, onde se observa que cada um deles foca em aspectos diferentes da GC.

A metodologia proposta por Wiig [16], lista os principais blocos para construção de GC: 1) Definição da gerência; (2) Pesquisa e mapeamento do conhecimento; (3) Planejamento estratégico do conhecimento; (4) Criar e definir conhecimentos relacionados com alternativas e iniciativas; (5) Retratar benefícios das expectativas das iniciativas GC; (6) Definir prioridades de GC; (7) Determinar requisitos essenciais de conhecimento; (8) Adquirir conhecimentos fundamentais; (9) Criar programas de transferência de conhecimentos integrados; (10) transformar, distribuir e aplicar 
conhecimento ativos; (11) Estabelecer e atualizar infra-estrutura de GC; (12) Gerênciar conhecimentos ativos; (13) Construir programas de incentivo; (14) Coordenar as atividades de GC a nível empresarial; (15) Facilitar o gerênciamento focado no conhecimento; (16) Monitorar a GC. Monsanto [17], construiu sua abordagem de GC baseada em cinco processos: (1) Ligação de pessoas com outras pessoas que possuem conhecimento; (2) Conectando as pessoas com informações; (3) Possibilitar a conversão de informações para conhecimento; (4) Encapsular conhecimentos, a fim de torná-lo mais fácil a transferência. (5) Difundir o conhecimento em torno da empresa.

Liebowitz [18], trouxe uma abordagem de GC com nove passos: (1) Transformar a informação em conhecimento; (2) Identificar e verificar o conhecimento; (3) Captação e proteção conhecimento. (4) Organizar os conhecimentos. (5) Obter e aplicar conhecimentos; (6) Combinar conhecimento. (7) Criar conhecimento; (8) Aprender conhecimentos; (9) Distribuir e vender conhecimento.

Uma metodologia que teve a preocupação de resolver limitações citadas anteriormente principalmente com relação aos pré-requisitos de GC foi a metodologia Smartvion [7]. que para cada fase especifica são definidos os respectivos procedimentos, sub-procedimentos e sua saída esperada. Suas fases são: estratégia, modelagem, ação, revisão e transferência, entretanto como o próprio autor relata que embora seja feita a diferenciação entre o conhecimento tácito e explicito o endereçamento de cada tipo não é abordado pela metodologia.

A metodologia COMMONKADS, [15] [19], consiste em três componentes principais: o primeiro, propõe uma modelagem para representar e analisar tarefas e o respectivo domínio do conhecimento, os agentes e os aspectos da comunicação das tarefas executadas por diferentes agentes. O segundo, oferece um conjunto de tarefas modelo que podem ser utilizados como aproximações iniciais de tarefas específicas em um determinado domínio de interesse. O terceiro, provê um conjunto de atividades para o desenvolvimento de sistemas de software no contexto global de uma organização, seus processos atuais, e as suas necessidades. Nesta metodologia, os modelos citados são validados através de simulação em papel ou através de protótipos.

A metodologia Proposta por [20] utiliza princípios e fases do planejamento interativo para aplicação de gerência do conhecimento no suporte a pesquisa cientifica e é considerada como uma metodologia de base criativa para resolver problemas. No planejamento interativo existem cinco fases: (1) formulação do problema, (2) fim do planejamento, (3) planejamento médio, (3) planejamento dos recursos, (4) concepção de implementação e (5) controle. Estas fases devem ser consideradas como um processo sistêmico. Onde cada fase pode ser iniciada em qualquer ordem. Após a apresentação de conceitos como: conhecimento, tipos de conhecimento, modelagem e metodologias, o item 2.5, a seguir faz uma relação entre e educação tecnológica e GC, mostrando como esta pode colaborar com os processos de aprendizagem da ET.

\section{EDUCAÇÃO TECNOLÓGICA E GERÊNCIA DO CONHECIMENTO}

A rapidez das mudanças no mundo, especificamente, as atualizações da tecnologia refletem especialmente nas mudanças do trabalho, exigindo desafios como qualificação e atualização de profissionais de uma forma cada vez mais ágil, sobretudo tratando-se de um profissional da área tecnológica. Esta atualização tecnológica exige novas habilidades, novos perfis e novos papeis profissionais exigindo sempre a união de teoria e prática [8] [9]. Neste contexto a educação tecnológica, participa de uma forma crucial provendo o ensino das ferramentas relacionadas com cada tecnologia, que vai alem do mero conhecimento de suas características chegando ao ponto de preparar o aluno através do desenvolvimento de habilidades e capacidades. 
No Brasil, de acordo com a proposta do Ministério da Educação Brasileira (MEC), a educação profissional e tecnológica deve estar integrada às diferentes formas de educação, ao trabalho, à ciência e à tecnologia. Esta política pública é suportada por uma vasta legislação [21], que abrange, leis, portarias, decretos e pareceres, dentre eles o parecer [22] que trata das diretrizes curriculares nacionais para a educação profissional e tecnológica. Este parecer visa o desenvolvimento sustentável do país comprometido com a redução das desigualdades sociais, através de referencias como a certificação de conhecimentos e de competências. Para um melhor entendimento do que é a educação tecnológica é importante esclarecer que tecnologia envolve um conjunto organizado e sistematizado de diferentes conhecimentos científicos, empíricos e até intuitivos voltados para um processo de aplicação na produção e na comercialização de bens e serviços. [23]. Neste contexto a educação profissional e tecnológica visa garantir ao cidadão o permanente desenvolvimento de aptidões para a vida produtiva e social.

O processo de aprendizado é também um processo de comunicação, e esta pode ser facilitada pela GC que pode colaborar sinergicamente na transferência do conhecimento pelo uso de recursos como pessoas, tecnologias e processos. Esta transferência é ainda mais crítica no contexto da educação tecnológica pela rapidez necessária para sua atualização e pela quantidade de informações necessárias para a mesma, exigindo assim dos professores um constante e considerável investimento de tempo para manter-se atualizado. Um aspecto importante também, segundo [24], é a preocupação com a limitação da capacidade do professor de transmitir a próxima geração níveis de ensino inferiores ou iguais ao seu, em outras palavras, um professor com um nível de habilidades e conhecimentos mensurados em x pode ensinar à próxima geração de competências apenas até x.

A GC pode colaborar com a administração desta limitação através de seus processos de modelagem e aplicação do conhecimento que serão abordados na possível metodologia apresentada neste trabalho. Nesta perspectiva, de desafio de comunicação, o E-learning pode entrar em cena como um caminho pára auxiliar nesta limitação e desafio de comunicação. E-learning e gestão do conhecimento possuem um foco semelhante: como melhorar conhecimento e sua utilização dentro das organizações. A gestão do conhecimento pode contribuir de forma estratégica na concepção de sistemas de E-Learning adaptativos, focados nas habilidades cognitivas individuais, fornecendo a infra-estrutura para o uso do conhecimento no processo de aprendizagem através de processos como: captura, transferência, entrega e mensuração do conhecimento. Outro aspecto da GC é fornecer um conteúdo adaptativo em ambientes de aprendizagem colaborativos podendo assim incrementar a eficácia da aprendizagem [25]. O item 2.6, a seguir, explica os objetivos e aplicações dos objetos de conhecimento.

\section{OBJETOS DE CONHECIMENTO.}

O objetivo principal do objeto de conhecimento (OC) é fazer uma abordagem conceitual e pratica do conhecimento através da separação entre seu conteúdo e forma, um OC é separado das estratégias usadas para apresentar, utilizar ou testa-lo, podem ser combinados em estruturas de conhecimento (modelos mentais cognitivos) responsáveis por representar modelos. É constituído por um conjunto de campos (contentores) com os componentes de conhecimento necessários para implementar uma variedade de estratégias instrucionais, estes componentes incluem: nome, descrição, valores e representações correspondentes para as propriedades da entidade. Esta composição permite que os OC sejam relacionados para formar o espaço de conhecimento, composto pelos OC que determinam o domínio da aplicação [26]. No próximo item, é discutida a visualização do conhecimento e sua participação na ET. 


\section{VISUALIZAÇÃO DO CONHECIMENTO}

A visualização do conhecimento, segundo [27], amplifica e acelera a sua cognição. Pode ajudar a educação tecnológica de várias formas como: facilitar a cognição do conhecimento complexo e de grandes quantidades de informação. A combinação de sistemas de informação com capacidades cognitivas humanas, tais como encontrar padrões, e a utilização da visualização do conhecimento como uma interface entre os dois é possível potencializar o processo cognitivo humano. Este processo é dificultado pela assimetria do conhecimento que expressa a diferença no nível de compreensão de determinado assunto entre duas ou mais pessoas [28]. A seguir são apresentadas as relações entre GC, Web Semântica e também como seus conceitos podem colaborar com a educação.

\section{GERÊNCIA DO CONHECIMENTO, WEB SEMÂNTICA E EDUCAÇÃO, CONCEITOS E RELAÇÕES.}

A internet, segundo a visão de [29], foi concebida originalmente para funcionar de uma forma semântica, através da incorporação de significado no seu conteúdo permitindo a interação entre humanos e máquinas. O que representaria uma Web que poderia evoluir para um estado em que o raciocínio da máquina será onipresente e teria um poder devastador. Esta Web semântica seria responsável por habilitar varias tecnologias para disseminação e comunicação do conhecimento como: comunidades de pesquisa, banco de dados, sistemas inteligentes, engenharia e representação do conhecimento, recuperação da informação e inteligência artificial [13]. Poderia também melhorar o desempenho de sistemas de aprendizado relacionados com ET, resolvendo a questão do correto aproveitamento da abundancia de dados e a alta escala e disponibilidade de documentos (ferramentas web 2.0).

As redes sociais possuem um papel importante na medida em que suportam interações, explicando assim o papel privilegiado da web semântica como uma base de apoio a essas redes, em especial com participantes distribuídos geograficamente [30]. Outro aspecto importante para a web colaborar no ensino da tecnológica é a necessidade de conteúdo inteligente (web inteligente) e semanticamente preparado para o desenvolvimento e uso de objetos de aprendizagem e documentos ativos. Estes conteúdos seriam implementados através de ontologias que usariam recursos de semântica para sua devida interação com a aprendizagem e GC [31]. Neste contexto as ontologias podem exercer um papel de viabilizar a recuperação e uso do conhecimento. Entretanto, chamam atenção para o fato de que somente a implementação de web semântica e seu respectivo processamento dos dados, por parte das maquinas, não é suficiente para a transformação de conhecimento a partir de dados [31].

O foco da ET é a interação enquanto na GC o foco é a qualidade dos dados, estas perspectivas estão fortemente interligadas. A GC pode melhorar a qualidade dos dados disponibilizando condições para efetivo uso de representação do conhecimento para web semântica. Dentre estas se destacam: a modelagem de contexto de domínio, portabilidade do OC, gerenciamento de mudanças que são de estrema importância porque são responsáveis pela interação entre os focos de ET e GC. Entretanto, como essas estruturas de componentes semânticos iriam colaborar de uma forma construtiva com a ET? Segundo [31], a semântica pode auxiliar a educação tecnológica participando na resolução de problemas como: desambiguação, definição de contexto e meta-informação, onde a resolução destes problemas trará melhorias em duas dimensões principais: qualidade dos dados e qualidade de interação.

A interação destes dados em um ambiente de ET de forma que possam ser utilizados trata-se de um 
processo de captura de conteúdo. Os dados semânticos, ou dados semanticamente enriquecidos, são dados combinados com software que permitam a contextualização através de metadados, dados semânticos ou dados compreensíveis. O modelo de interação é pensado em termos de entrega de conteúdo e conhecimento para compor um plano concreto de representação do conhecimento de forma semântica. Um exemplo de oportunidade de uso deste conhecimento semântico seria a abundância crescente de dados oferecida pela Web e sua alta disponibilidade de documentos eletrônicos. Após a apresentação dos fundamentos relacionados com o trabalho a seção 3, a seguir explica como surgiu a idéia de concepção da metodologia e como foi desenvolvida.

\section{METODOLOGIA DA PESQUISA}

A idéia para construção da metodologia, surgiu a partir de reflexões relacionadas com uma melhor forma de lidar com o conhecimento no contexto de ensino. Como por exemplo: de que forma gerenciar o conhecimento a ser utilizado em um curso? E, de que forma esta gerência do conhecimento seria implementada? A metodologia foi desenvolvida de forma progressiva através dos seguintes passos: inicialmente foram identificados quais os processos de GC que participariam da mesma, em seguida, estes processos foram distribuídos em fases. Cada fase da metodologia foi implementada através de um protótipo com o objetivo de construir provas conceituais para a validação e desenvolvimento das idéias.

\section{ESTUDO DA VIABILIDADE}

A seguir, são apresentados os fundamentos de uma metodologia, que tem como objetivo principal demonstrar a viabilidade de utilização da GC na educação tecnológica. Este objetivo é alcançado pela utilização de processos da GC como criação, recuperação, transferência, aplicação e visualização. Os processos, juntamente com o conteúdo dos cursos, são modelados e implementados, em uma etapa posterior ocorre uma avaliação onde os conteúdos que foram implementados através dos OC sofrem uma validação, ocorrendo na seqüência à atualização da base do conhecimento, que é implementada através de ontologias. A apresentação da metodologia é feita inicialmente através de seus componentes principais e em seguida são apresentadas às fases e seus respectivos processos, e para um melhor entendimento é mostrada a estrutura de uma ontologia participante do processo.

\section{COMPONENTES}

A figura 2, a seguir, apresenta os componentes principais da arquitetura: objetos de conhecimento, avaliação, pessoal e processos.

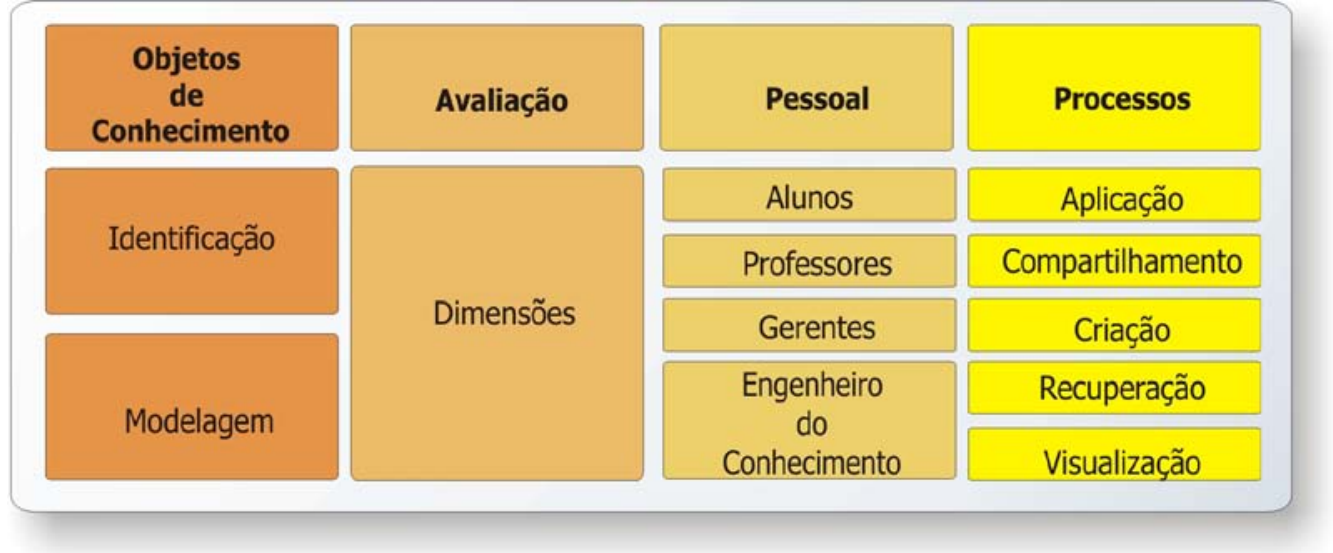

Figura 2 - Componentes Principais 


\section{OBJETOS DE CONHECIMENTO}

Os objetos de conhecimento descrevem de forma precisa o conteúdo das matérias e disciplinas a serem ensinados, sendo responsáveis por permitir a manipulação do conhecimento de uma forma ágil, para esta manipulação, no contexto do paradigma da GC e Web semântica. É necessário que este objeto do conhecimento tenha algumas propriedades como: representação do domínio do conhecimento, capacidade de receber inferências, capacidade de ser localizado, atualizado e transferido. Além dessas características, o OC pode também prover a organização de recursos relacionados como: áudio, vídeo, documentos, gráficos e etc.

A ontologia exemplificada foi construída através do editor de ontologias Protégé [32], que é um framework de código aberto construído em Java [33] para criar e gerenciar ontologias. O Editor Protégé, também faz automaticamente a geração do código em OWL (Web Ontology Language) [34], que é uma linguagem para definir e instanciar ontologias na Web. Um exemplo prático que valida esta modelagem seria um processo que examinaria este objeto de conhecimento, no nível de classe, permitindo com a ajuda de ferramentas de inferência saber de forma praticamente instantânea que conteúdos de determinada disciplina estão passiveis de receber atualização.

A figura 3, a seguir, que se trata de uma amostra reduzida, ilustra um OC que é modelado através de uma ontologia composta por um conjunto de classes e suas respectivas heranças. Esta modelagem permite separar o conhecimento de sua estrutura, a idéia principal é modelar o OC de forma que ele possa ser conhecido e contextualizado de forma ágil. No exemplo da figura abaixo pode se observar a classe domínio responsável pela contextualização citada e suas subclasses, competências, habilidades, disciplina, curso e assunto. O OC completo pode ser visto no Anexo.

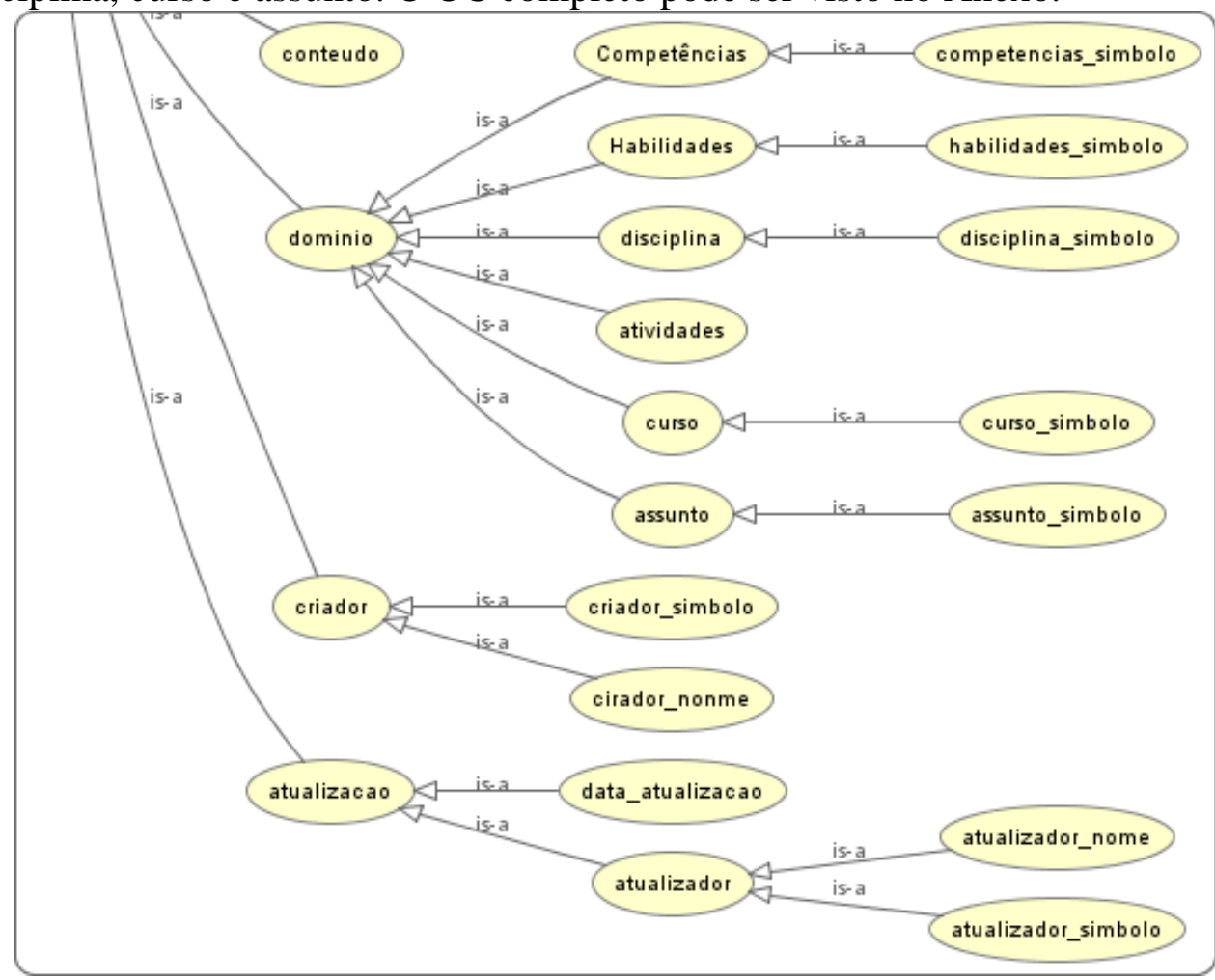

Figura 3 - Amostra: Classes da Ontologia Objetos de Conhecimento. 


\section{AVALIAÇÃO}

A avaliação tem como objetivo fundamental analisar se os objetos de conhecimento estão colaborando de forma satisfatória com o processo de aprendizagem e como podem ser melhorados para evoluírem com e tempo. Outra importante função da avaliação é examinar a qualidade do conteúdo utilizado, para atingir esses objetivos a avaliação será modelada a partir de um conjunto de dimensões e que serão traduzidas em forma de uma ontologia. A modelagem de Dimensões como: acesso, representação, nível de relevância, espaço de conhecimento, qualidade do conhecimento, qualidade das referencias e validade permitem conhecer o objeto do conhecimento de forma precisa. Cada dimensão examina o mesmo em uma perspectiva diferente e conforme os resultados da avaliação, os objetos do conhecimento podem receber atualizações e melhorias com o tempo. A freqüência da avaliação pode ser determinada a partir de necessidades específicas de cada curso, como por exemplo: a inserção de um novo professor colaborador ou até mesmo a inclusão de novos cursos e suas respectivas disciplinas.

\section{PESSOAL}

O processo de aprendizagem pode ser compreendido tambem, na perspectiva da GC, como um processo de transferência do conhecimento ente pessoas, para tanto o conhecimento precisa ser organizado e disponibilizado para os usuários finais do conhecimento que são alunos e professores. A princípio são identificados quatro participantes do processo: alunos, professores, gerentes e o engenheiro do conhecimento, onde cada componente participa de fases e atividades especificas no processo de implementação da GC. A seguir uma breve apresentação de cada participante.

- Alunos: Os alunos juntamente com os professores serão os usuários do conhecimento, possuem características próprias e importantes na perspectiva da GC como: diferentes capacidades cognitivas, motivações e envolvimento.

- Professores: São responsáveis por administrar a transferência do conhecimento aos alunos, e também por modelar e atualizar, juntamente com o engenheiro do conhecimento os objetos de conhecimento.

- Gerentes: Sua principal responsabilidade é controlar os processos de avaliação e analise do conhecimento, definindo quando deve ocorrer a avaliação e providenciando que atualizações e correções devem receber os objetos de conhecimento.

- Engenheiro do Conhecimento: Será o responsável pela modelagem e projeto dos objetos de conhecimento em conjunto com o professor, além de, modelar os processos de manipulação do conhecimento e modelar a avaliação.

\section{PROCESSOS}

Segundo [5], os processos de GC podem ser classificados em dois níveis: operacional e estratégico (ou tácito). No nível operacional tem-se os processos básicos de criação, armazenagem, transferência e utilização do conhecimento. Enquanto no nível estratégico respectivamente: planejamento, modelagem e controle do conhecimento. Na metodologia proposta os processos serão responsáveis por implementar a GC, são divididos em dois tipos: processos operacionais e processos estratégicos, a figura 4 a seguir, ilustra os processos e sua respectiva classificação. 


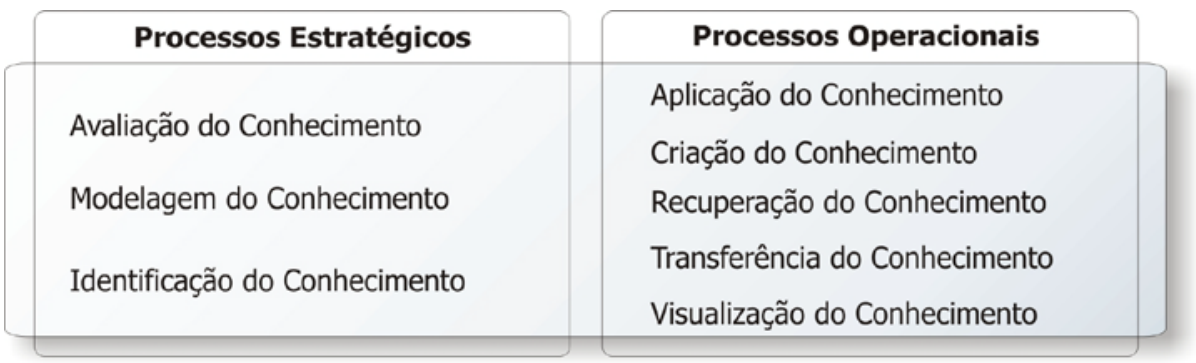

Figura 4 - Processos Operacionais e Processos estratégicos

Os processos estratégicos são responsáveis por mapear os conceitos relacionados com o conhecimento e também avaliar como os mesmo estão colaborando, no decorrer do tempo, com o processo de aprendizagem, os processos:

- Identificação do conhecimento: Neste processo são determinados os conceitos gerais relacionados com o domínio do conhecimento, e que serão os precursores dos objetos de conhecimento. É o momento também de relacionar os especialistas capacitados a colaborar com a composição do objeto do conhecimento;

- Modelagem do conhecimento: Após a identificação dos conceitos gerais, ocorre à modelagem dos objetos de conhecimento, os especialistas ou o especialista responsável deste objeto vão determinar as classes responsáveis, sua respectiva estrutura e hierarquia que vai ser responsável por representar o objeto em questão;

- Avaliação do conhecimento: É o processo que tem a responsabilidade de garantir o desenvolvimento constante da qualidade dos objetos de conhecimento, o gerente do conhecimento deve em primeiro lugar identificar os aspectos do OC que devem ser avaliados e definir a freqüência desta avaliação.

Os processos operacionais caracterizam-se por organizar e manipular o conhecimento que já foi identificado e modelado. A seguir uma breve descrição destes processos:

- Criação do conhecimento: É um processo operacional que tem a função de recolher o conhecimento para posterior recuperação e aplicação, deve garantir que o conhecimento armazenado no OC específico, no formato de ontologias, possa ser transferido e utilizado quando necessário.

- Transferência do conhecimento: Este processo tem o objetivo de garantir que o conhecimento armazenado possa ser disponibilizado a todos os participantes.

- Visualização do conhecimento: Este processo tem o objetivo de facilitar o acesso ao conhecimento, proporcionando uma forma alternativa de acesso ao seu conteúdo, outro benefício é auxiliar o trabalho do professor ao lidar com diferentes capacidades cognitivas, este processo é implementado através da associação de símbolos com determinadas classes onde estes símbolos representariam seu significado.

- Recuperação do conhecimento: O conhecimento armazenado precisa ser recuperado para sua posterior utilização, para isso este processo deve prover os meios para que o conhecimento possa ser localizado de acordo com suas características representadas.

- Aplicação do conhecimento. A intenção deste processo é facilitar e permitir a utilização do conhecimento nas atividades educacionais, desta forma este processo ao ser 
implementado deve responder as seguintes questões: como o OC vai ser utilizado nas atividades educacionais? Que atividades, documentos, disciplinas e cursos devem e podem participar este objeto?

\section{FASES}

A implementação da possível metodologia de GC ocorre através de um conjunto de oito fases, compostas por um conjunto de atividades, cada fase tem objetivos e artefatos específicos que serão utilizados nas fases seguintes, a figura 4, a seguir, ilustra o conjunto das fases e sua sinergia no decorrer do tempo.

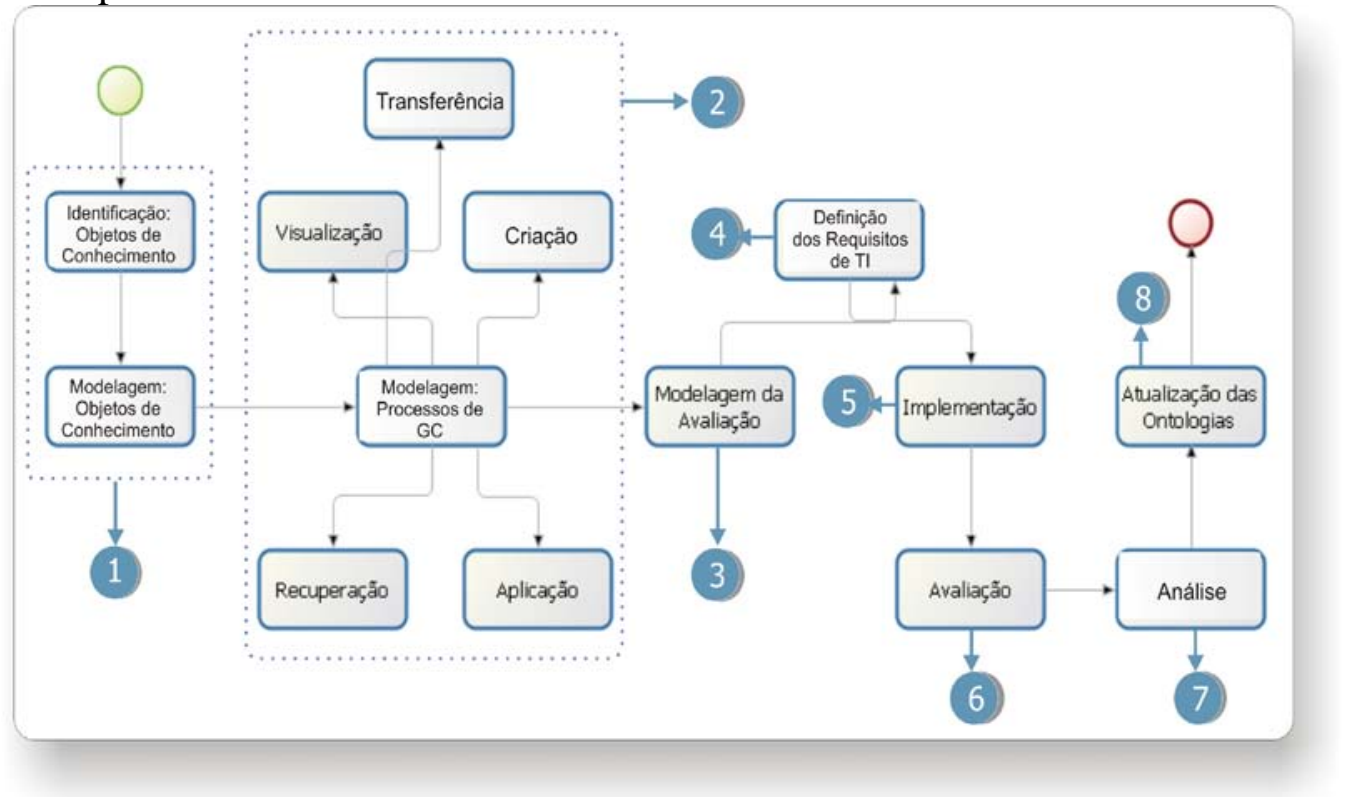

Figura 5 - Fases e Processos.

A seguir, uma breve descrição das fases ilustradas na figura 5.

Fase (1), Modelagem dos Objetos de Conhecimento. É a fase inicial, nela os objetos de conhecimento são identificados e modelados, os artefatos resultantes dessa fase são os objetos de conhecimento e toda a sua estrutura hierárquica de classes.

Fase (2), Modelagem dos processos do conhecimento. Este conjunto de atividades estruturadas será responsável por estabelecer as bases para a gerência do conhecimento. Podem ocorrer de forma concomitante e podem ser iniciados sempre que houver a manifestação da necessidade.

Fase (3), Modelagem da Avaliação. Esta modelagem tem o objetivo de prover um exame da qualidade dos objetos de conhecimento e seus respectivos conteúdos. Esta qualidade é observada pelo seu nível de colaboração na aprendizagem além de aspectos como referencias e atualização.

Fase (4), Definição dos requisitos de tecnologia da informação (TI). Os requisitos da estrutura de TI, hardware e software, devem ser projetados para atender a sinergia provocada pelos processos do conhecimento citados na fase (2).

Fase (5), Implementação. Nesta etapa ocorre à codificação dos processos de conhecimento, a disciplina de engenharia de software [35], vai ser a responsável por materializar os processos do conhecimento aplicados aos objetos de conhecimento modelados na fase (1) e (2), respectivamente. 
Fase (6), Avaliação. O gerente responsável pela gerência do conhecimento vai ser responsável por definir quando a avaliação será realizada, eventos como: novos cursos, novas disciplinas ou novos colaboradores podem ser determinar a aplicação de uma avaliação.

Fase (7) Analise. Após a aplicação da avaliação o gerente pode considerar um conjunto de aspectos disponíveis, em forma de classes e suas hierarquias, para examinar o objeto do conhecimento, pode, por exemplo, através de inferências selecionar objetos que estão desatualizados ou com atualização inferior a uma faixa definida.

Fase (8), Atualização. A análise vai fornecer os subsídios para aplicar as alterações e atualizações necessárias nos objetos de conhecimento. Fechando o conjunto de fases do processo.

\section{DEMONSTRANDO UM PROCESSO}

Com o objetivo de demonstrar a utilização da metodologia em um caso prático foi construído um protótipo. Este protótipo foi desenvolvido de acordo com a disciplina de Engenharia de Software (ES), que foi responsável por fornecer os aspectos necessários para sua produção [35]. Através dos processos de software, a ES, provê um conjunto estruturado de atividades para desenvolver um sistema de software.

A linguagem de modelagem UML (Unified Modeling Language) [36] foi utilizada para descrever o projeto especificado pela ES, através de modelos que representam, de uma forma idealizada, o software a ser construído. Estes modelos permitem um melhor gerenciamento da complexidade e uma melhor comunicação entre os participantes do processo de desenvolvimento. A composição dos diagramas com a linguagem UML antecedeu a criação do protótipo que foi utilizado para implementar os processos citados anteriormente, especificamente na fase de elaboração do protótipo. A figura 6, a seguir, ilustra através do diagrama de casos de uso da UML, as funções do usuário professor no sistema, funções estas que correspondem às atividades do professor.

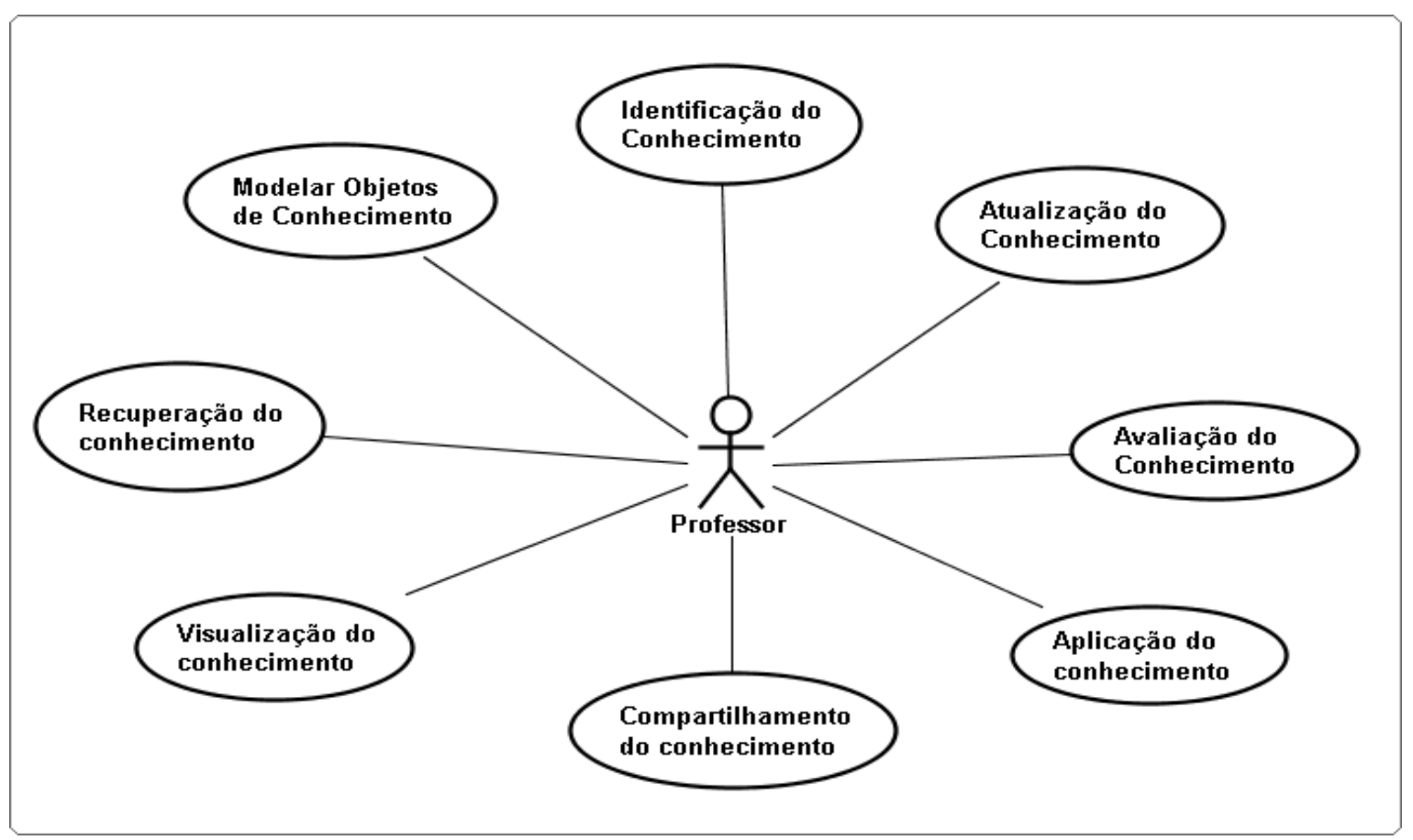

Figura 6 - Casos de Uso: Professor. 
A seguir, é ilustrado um processo crítico deste trabalho que é a criação do objeto de conhecimento. conforme ilustrado na figura 7, a criação do conhecimento ocorre pela composição das dimensões modeladas anteriormente na fase de modelagem.

A figura 7, a seguir, ilustra a primeira etapa da composição do objeto de conhecimento que é a identificação, que ocorre através das seguintes informações: criador que será o responsável pela composição do OC, nome que identificará o OC e a descrição, que é um breve resumo do objeto em questão.

\begin{tabular}{|c|c|c|}
\hline 多 & $\square[$ & $\mathrm{x}$ \\
\hline \multicolumn{3}{|c|}{$\begin{array}{l}\text { Processo: Criação do Conhecimento } \\
\text { Identificação }\end{array}$} \\
\hline \multicolumn{3}{|c|}{ Etapa 1 de 7: Identificação } \\
\hline Criador & Vitoria Regia| & \\
\hline Nome & Conceitos de Processo de Software & \\
\hline \multirow[t]{2}{*}{ Descrição } & Este objeto de conhecimento descreve o contreudo de processo de sof & \\
\hline & Próximo & \\
\hline
\end{tabular}

Figura 7 - Identificação do OC.

Na figura 8, a seguir, através de um protótipo é ilustrada a segunda etapa da composição do OC que é o domínio do objeto do conhecimento formado respectivamente por: curso disciplina, atividades, competências e habilidades. Cada dimensão possui um conjunto de elementos que por sua vez são classes da ontologia objeto de conhecimento, este conjunto de classes descreve o conteúdo do OC.

\begin{tabular}{|l} 
Processo: Criação do Conhecimento \\
Identificação/Dominio
\end{tabular}




\section{Figura 8 - Protótipo: Criação do Conhecimento.}

\section{CONCLUSÃO}

A GC está em pleno desenvolvimento, necessitando ainda de mais pesquisa e análise principalmente no campo das metodologias para sua efetiva implementação, entretanto já dispõe de recursos para suportar um melhor uso do conhecimento nas organizações e instituições de ensino. A metodologia proposta por este trabalho, contribui para a GC ao permitir uma utilização de seus paradigmas de uma forma clara através de suas definições de processos e fases.

A abordagem semântica do conhecimento proposta pela metodologia traz uma implicação importante que é facilitar a sua manipulação. Com esta abordagem, o conhecimento ao ser modelado é expresso de forma mais completa e traz benefícios como: melhor acesso, discussão e maior facilidade para ser compartilhado. Este trabalho contribui para ET ao propor um tratamento e formalização do conhecimento a ser utilizado ao longo do tempo nos conteúdos de cursos e disciplinas, contribui também com o controle dos processos de manipulação do conhecimento como criação, recuperação aplicação, transferência e visualização do conhecimento. O controle destes processos pode auxiliar nas atividades de aprendizagem da ET, principalmente a transferência do conhecimento, colaborando assim com a superação dos desafios inerentes da ET como: a rápida atualização de conteúdos e a grande quantidade de informações necessárias no processo de aprendizagem da educação tecnológica.

Uma limitação observada neste trabalho é a falta de uma descrição mais detalhada dos processos e suas respectivas atividades, que poderiam ser representados através de categorias específicas de ontologias conhecidas como ontologias de atividades. Outro aspecto que pode ser aprimorado é o processo de visualização do conhecimento, que embora tenha recebido um tratamento inicial através da composição de símbolos em determinadas classes dos OC, conforme anexo, ainda é pouco para o potencial que este processo possui principalmente relacionado com os aspectos de cognição e assimetria do conhecimento citados.

Este trabalho está em processo de expansão através das seguintes abordagens: expansão da metodologia em desenvolvimento para pesquisa cientifica; manipulação de meta conhecimento no contexto da educação tecnológica; visualização do conhecimento e mapeamento do conhecimento tácito.

\section{REFERÊNCIAS BIBLIOGRÁFICAS}

1. TOCHTERMANN, K., H, MAURER: Knowledge Management and Environmental Informatics. Journal of Computer Science 6, 5. 517-536 (2000).

2. LYTRAS, M.: Knowledge management strategies : a handbook of applied technologies, Hershey, New York, 2008.

3. BASKSHI, R.: Key Issues in Knowledge Management: Introductory Analysis, Pakistan Journal of Social Sciences, 2005.

4. LYLES, S. et al: Organizational learning and knowledge management: Agendas for future research. In Easterby-Smith, M. and Lyles, M. (Eds.) Blackwell handbook of organizational learning \& knowledge management (pp. 639-666). Oxford, UK: Blackwell Publishing , 2003.

5. NICOLAS, P.: A Hieraquical Model for Knowledge Management, ESSEC Business School, France, Encyclopedia of Knowledge Management. Hershey Hershey - USA : Idea Group Reference, 2006. 
6. BURES V.: KM-BEAT-IT: Methodology of Knowledge Management Implementation, University of the Czech Republic, 2005.

7. MONTANO, R. et al: SMARTVISION: A knowledge-management methodology. The Journal of Knowledge Management 5.4 (2001): 300-310. 2001.

8. OlIVEIRA, G. A Educação Profissional Integrada ao Ensino Médio No Paraná: Avanços E Desafios, 2009.

9. BASTOS, A.V., DULTRA, J.A.: Trabalho, qualificação e competências: um desafio atual. Revista Brasileira de Docência, Ensino e Pesquisa em Administração, Vol. 1, n. 2, Julho/2009

10. EUROPEAN COMMITTEE FOR STANDARDIZATION, European Guide to good Practice in Knowledge Management - Part 5: KM Terminology, 2004. disponível em: http://www.knowledgeboard. com/item/637, accesso em 11 de Julho de 2009.

11. HEY, J.: The Data, Information, Knowledge, Wisdom Chain: The Metaphorical link. 2004. Disponível em: http://ioc.unesco.org/oceanteacher/ oceanteacher2/02_InfTchSciCmm/ DIKWChain.pdf . Acesso em: 12/12/2008.

12. EIN-DOR, P.: Taxonomies of Knowledge management, Encyclopedia of Knowledge Management. Hershey - USA : Idea Group Reference. 2006.

13. SIMON, K., MALCOLM, C.: An Introduction to Knowledge Engineering, Springer-Verlag New York, NJ, USA, 2006.

14. FENSEL, D.: Ontologies: A silver bullet for knowledge management and electronic commerce. Berlin/Heidelberg/ New York: Springer. 2001.

15. MOTELEB, A.A., WOODMAN, M. “Uncovering a KMSD Approach from Practice.” The Electronic Journal of Knowledge Management, Volume 7, Issue 1, 2009, pp. 123 - 134, disponível em: http: www.ejkm.com. acesso em: 10 de Julho de 2008.

16. WIIG, K. M.. Knowledge Management Foundations: Thinking about Thinking -How People and Organizations Create, Represent, and Use Knowledge, Arlington, TX: Schema Press. (1993)

17. JUNNARKAR, B., Creating fertile ground for knowledge management at Monsanto, Perspectives on Business Innovation 1, 1999, disponível em: http://www.businessinnovation.ey.com/journal/issue1,acesso em 06 Maio de 2009.

18. LIEBOWITZ, J., Building Organizational Intelligence: A Knowledge Management Primer, CRC Press, Boca Raton, FL, 2002.

19. SCHREIBER, G., et al: Knowledge Engineering and Management: The CommonKADS Methodology. Boston Mass: MIT Press. 1999.

20. MA, T. et al: Roadmapping as a way of knowledge management for supporting scientific research in academia, Systems Research and Behavioral Science, 2006

21. SETEC, Educação profissional e tecnológica: legislação básica - Rede Federal / Secretaria de Educação profissional e tecnológica. 7. ed. Brasília: MEC, SETEC, 2008.

22. CONSELHO NACIONAL DE EDUCAÇÃO (BR). Parecer CNE/CEB n ${ }^{\circ} 16$ de 26 de novembro de 1999. Diretrizes Curriculares para Educação Profissional de Nível Técnico. Diário Oficial da União, Brasília, 5 out. 1999., Disponível em: http://portal.mec.gov.br/setec/arquivos /pdf/PCNE_CEB16_99.pdf. acesso em 21 de Agosto de 2008

23. GRINSPUN, Z. Educação Tecnológica. São Paulo. Cortez (1999)

24. RIGOLINI, J.: Education technologies, wages and technological progress, Journal of Development Economics 75 (2004) 55- 77. 2003.

25. XU, D., WANG, H.: Integration of Knowledge Management and E-Learnig, Encyclopedia of Knowledge Management. Hershey Hershey - USA : Idea Group Reference, 2006. 
26. GOU, J., et al: An Ontology Based Knowledge Clustering Method in Knowledge Space, College of Information Science and Engineering, Huaqiao University, 362021 Quanzhou, China, 2007.

27. S.-O. TERGAN., T. KELLER: Knowledge and Information Visualization, LNCS 3426, pp. 1 - 23, 2005. (C) Springer-Verlag Berlin Heidelberg 2005.

28. SHARMA, P., FIEDLER, S.: Supporting self-organized learning with personal webpublishing technologies and practices. Journal of Computing in Higher Education, 18(2), 3-24. (2007)

29. TIM BERNERS-LEE, J. HENDLER, O. LASSILA. The Semantic Web. Scientific American. http://www.scientificamerican.com/2001/0501issue/0501berners-lee.html, May 2001, p. 29-37. acesso em 10 de Dezembro de 2009.

30. CHI, E.H. The Social Web: Research and Opportunities, Computer Volume 41, Issue 9, Sept. Page(s):88 - 91 Digital Object Identifier 10.1109/MC.2008.401, EEE, 2008.

31. KOHLHASE E KOHLHASE: Semantic Knowledge Management for Education, Proceedings of the IEEE. Vol. 96, No. 6, June 2008.

32. STANFORD MEDICAL INFORMATICS, Protégé, 2009. disponível em: URL: http://protege.stanford.edu. Acesso em: 19 de Novembro de 2009.

33. SUN. Java technology Home Page. 2006. Disponível em http://java.sun.com/, acesso em julho de 2009.

34. W3C, OWL Web Ontology Language, dispinível em < http://www.w3.org/TR/owl-features/>, acesso em agosto de 2009.

35. IAN SOMMERVILLE: Software Engineering, 8a Edição. Addison Wesley (2007).

36. OBJECT MANAGEMENT GROUP, “UML 2.0 Superstructure Final Adopted Specification”, OMG document ptc/03-08-02, 2004. 


\section{ANEXO - Ontologia: Objeto do Conhecimento}

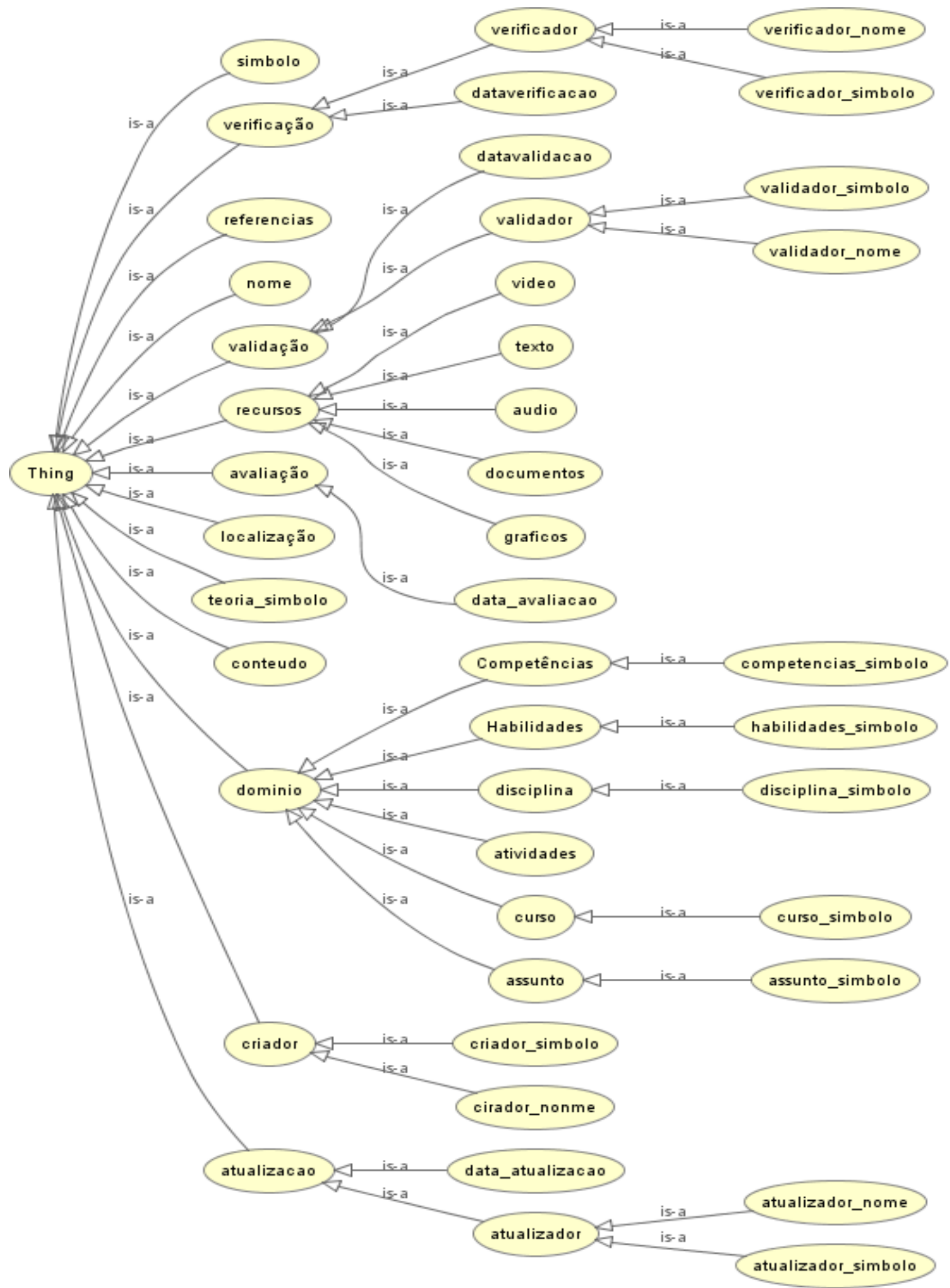

